\title{
Study of Driven Magnetic Reconnection in a Laboratory Plasma
}

\author{
Masaaki Yamada, Hantao Ji, Scott Hsu, Troy Carter, Russell Kulsrud, Norton Bretz, \\ Forrest Jobes, Yasushi Ono*, and Francis Perkins** \\ Plasma Physics Laboratory, Princeton University, P. O. Box 451, Princeton, NJ 08543
}

\begin{abstract}
The Magnetic Reconnection Experiment (MRX) has been constructed to investigate the fundamental physics of magnetic reconnection in a well controlled laboratory setting. This device creates an environment satisfying the criteria for a magnetohydrodynamic (MHD) plasma $\left(S>>1, \rho_{i}<<L\right)$. The boundary conditions can be controlled externally, and experiments with fully three-dimensional reconnection are now possible. In the initial experiments, the effects of the third vector component of reconnecting fields have been studied. Two distinctively different shapes of neutral sheet current layers, depending on the third component, are identified during driven magnetic reconnection. Without the third component (anti-parallel or null-helicity reconnection), a thin double-Y shaped diffusion region is identified. A neutral sheet current profile is measured accurately to be as narrow as order ion gyro-radius. In the presence of an appreciable third component (co-helicity reconnection), an O-shaped diffusion region appears and grows into a spheromak configuration.
\end{abstract}

PACS numbers: 52.30.Jb, 96.60.Rd, 94.30.Lr

DSTMIBUTION OF THIS DOCUMENT IS UNLMTRE

MASTER

Typeset using REVTEX 


\section{DISCLAIMER}

This report was prepared as an account of work sponsored by an agency of the United States Government. Neither the United States Government nor any agency thereof, nor any of their employees, make any warranty, express or implied, or assumes any legal liability or responsibility for the accuracy, completeness, or usefulness of any information, apparatus, product, or process disclosed, or represents that its use would not infringe privately owned rights. Reference herein to any specific commercial product, process, or service by trade name, trademark, manufacturer, or otherwise does not necessarily constitute or imply its endorsement, recommendation, or favoring by the United States Government or any agency thereof. The views and opinions of authors expressed herein do not necessarily state or reflect those of the United States Government or any agency thereof. 


\section{DISCLAIMER}

Portions of this document may be illegible in electronic image products. Images are produced from the best available original document. 


\section{INTRODUCTION}

Interplay between plasma and magnetic field is best manifested by magnetic reconnection, a topological rearrangement of magnetic field lines ${ }^{1-4}$. Magnetic reconnection is considered to be a key process in the evolution of solar flares ${ }^{5-9}$ and in the dynamics of the Earths magnetosphere $\mathrm{e}^{10-14}$. It also occurs as one of the relaxation processes in fusion research plasmas ${ }^{15,16}$, and it often plays a dominant role in determining the confinement characteristics of high-temperature fusion plasmas.

The study of solar flares has been intensified due to the availability of soft X-ray pictures of the sun recently taken by the Yohkoh satellite ${ }^{8}$. Solar flares provide a paradigm for physical systems where magnetic energy is stored in a force-free magnetic equilibrium configuration and then, via slow adjustment of an external condition, brought to a situation of ideal magnetohydrodynamic (MHD) instability which forms intense current sheets and initiates reconnection. The evolving solar flares ${ }^{17}$ are the best known example of rapid conversion of magnetic energy into heat. Recent theoretical work has focused on two-dimensional (2-D) models of the evolution of force-free magnetic arcades ${ }^{17,18}$ whose field-line feet are advected by flows in the solar photosphere. However, it has been recognized that in realistic boundary conditions the effects of the third magnetic field component plays an important role ${ }^{19}$.

The most common description of magnetic field line reconnection is shown in Fig. 1(a), on which the 2-D theory have been based ${ }^{20-23}$. In actual reconnection phenomena, such as in solar flares, the magnetosphere, and most laboratory experiments, the magnetic field lines have three vector components as illustrated in Fig. 1(b). When the third component $B_{T}$ vanishes in both plasmas [null-helicity case, Fig. 1(i)], the conventional 2-D reconnection is applicable. In the presence of a third component, (1) the field lines reconnect at an angle when uni-directional toroidal fields exist [co-helicity case, Fig. 1(ii)] or (2) they reconnect with anti-parallel geometry when the toroidal fields are oppositely directed [counter-helicity case, Fig. 1(iii)]. Note that the reconnecting field lines are anti-parallel for both null-helicity and counter-helicity merging. Although case (i) and case (iii) in Fig. 1(b) are similar in local 
description, they can be quite different in the global MHD picture.

Dayside reconnection in the terrestrial magnetosphere ${ }^{10-12}$ is depicted as a series of "flux transfer events" and has a strong dependence on the merging angles of field lines. It has been recognized that the third vector component plays an important role in the reconnection process $^{11,24}$. It has also been known that the merging angle of reconnecting field lines plays an important role in the dayside magnetopause: namely, southward solar winds (or IMFinterplanetary magnetic field) reconnect much faster with the earth's dipole field (northward) than northward $\mathrm{IMF}^{12,14}$.

Major and minor disruptions of tokamak plasmas for magnetic fusion research are caused by reconnection of magnetic field lines, resulting in degradation of confinement properties $^{16,25}$. It is rather difficult to monitor precise evolution of field lines in hot tokamak plasmas since it takes place in a high energy density environment which destroys probes and is unsuitable for local measurement of magnetic fields. Recently, evolution of the safety factor ( $q$-profile) has been measured by motional Stark effect (MSE) diagnostics ${ }^{26}$ assuming axisymmetry. During a sawtooth crash, magnetic reconnection has been verified as partial mixing of field lines, and the resulting small changes of the $q$ profile are documented. It is shown ${ }^{16}$ that the $q$ values stay substantially below unity despite the fact that the electron temperature contour evolution from electron cyclotron emission (ECE) diagnostics apparently show Kadomtsev-type full reconnection patterns. A simultaneous analysis of the electron temperature profile and $q$ profile evolutions has lead to the proposal of a model ${ }^{16}$ in which a non-axisymmetric, three-dimensional (3-D) MHD mode plays an essential role in driving field line reconnection. This mode forces reconnection to occur at the point of weakest magnetic field. Fast parallel transport along the newly connected field lines can cause a rapid influx of internal energy through the $\mathrm{X}$-point region and thus create a fast crash of the central plasma pressure.

The MRX device ${ }^{27}$ has been constructed to investigate the fundamental physics of magnetic reconnection in a well controlled laboratory setting. This device creates an environment satisfying the criteria for MHD plasmas, and the boundary conditions can be controlled 
externally. The primary objective of the MRX experiment is the fundamental study of reconnection utilizing a very flexible merging toroidal plasma configuration as portrayed in Figs. 2 and 3. The overall initial geometry is axisymmetric (and hence 2-D), but nonaxisymmetric configurations can also be formed to study 3-D characteristics of merging. These plasmas have high conductivity with large Lundquist number $S$ of approximately $10^{3}$ and ion gyro-radii smaller than the plasma scale length, permitting true MHD flows to develop.

Initial experiments on the merging of two annular plasmas have been carried out successfully on MRX. The goal of our experimental work is to create a number of different initial states and to study: (1) whether we can create the familiar axisymmetric 2-D reconnection layer and whether reconnection processes remain axisymmetric or spontaneously lead to 3-D phenomena (2) how the reconnection rate depends on the third component of merging fields (3) the dependence of the reconnection rate on global MHD forces which can be controlled by external boundary conditions (4) the MHD plasma flows associated with reconnection and their conversion to heat. Some of the above issues have been addressed in our initial experiments and in the TS-3 experiments at the University of Tokyo ${ }^{28-31}$. In all these areas, we believe the inter-relationship between the global magnetic configuration and the resistive reconnection region will play a key role in determining the reconnection features.

\section{PHYSICS ISSUES OF MAGNETIC RECONNECTION}

To describe the motion of magnetic field lines in a plasma, Eq. (1) for the evolution of the magnetic field $B$ can be derived by combining Maxwell equations and Ohms law:

$$
\frac{\partial B}{\partial t}=\nabla \times(v \times B)+\frac{\eta}{\mu_{0}} \nabla^{2} B
$$

The first term on the right-hand side represents motion of field lines frozen-in to the plasma ${ }^{\mathbf{1}}$. The second term describes diffusion of magnetic fields with the diffusion coefficient proportional to the plasma resistivity. The representative time scale for the second term, 
$\tau_{\mathrm{D}}=\mu_{0} L^{2} / \eta$, is called the diffusion time, where $L$ is the scale of the plasma, and $\eta$ and $\mu_{0}$ are the plasma resistivity and the vacuum permeability, respectively. If the Lundquist number is defined by $S \equiv \tau_{\mathrm{D}} / \tau_{\mathrm{A}}$, where $\tau_{\mathrm{A}} \equiv L / V_{\mathrm{A}}\left(V_{\mathrm{A}}\right.$ is the Alfvén speed), it must be significantly larger than unity in order for the plasma to be treated as a MHD fluid. For typical MHD plasmas such as solar flares, $S>10^{10}$, for tokamaks, $S>10^{7}$, and for the $\mathrm{MRX}^{27}$ and TS- $3^{28-31}$ experiments, $S \sim 10^{2}$ to $10^{3}$.

Ideal MHD deals with the physics of highly conducting $(\eta=0)$ fluids, where the approximation $E_{\|}=\boldsymbol{E} \cdot \boldsymbol{B} / B=0$ is valid. Physically, this approximation means that finite parallel currents can be drawn by vanishing small electric fields. When $E_{\|}=0$, one can prove $^{1}$ that magnetic field lines move with the fluid and remain intact. This corresponds to putting $\eta=0$ in Eq.(1). But ideal hydromagnetic flows can lead to singular current density sheets $^{20-23}$ where $E_{\|}$becomes sufficiently large so that a magnetic field line can lose its original identity and reconnect to another field line. The topology of the magnetic configuration changes and large $\boldsymbol{j} \times \boldsymbol{B}$ hydromagnetic forces often result.

Research on the fundamental physics processes of reconnection and their hydromagnetic consequences has been largely theoretical. Most theories of magnetic reconnection are based on steady-state, 2-D models with magnetic X-points and laminar flows. The SweetParker ${ }^{20,21}$, Petschek ${ }^{22}$, and Sonnerup ${ }^{23}$ models are well known. The extensive literature of 2-D theoretical research has remained unchallenged by a decisive MHD plasma experiment. Only a few recent laboratory experiments have created an MHD plasma environment in which the global boundary condition can be externally controlled, despite the fact that magnetic reconnection always occurs during plasma formation and/or configuration change and is regarded as the most important self-organization phenomena in plasmas. A series of experiments were carried out by Stenzel and Gekelman using a linear plasma device in which reconnection was induced by driving currents in parallel plate conductors ${ }^{32,33}$. The effects of the third field components were not studied in their experiments. Although the ion gyro-radius was too large and $S$ was too small for their plasmas to be fully in the MHD regime, thorough local measurements were made. The local force on the plasma $j \times B-\nabla p$ 
was compared with the measured particle acceleration. It was found that the ion acceleration was strongly modified by scattering of wave turbulence. Current sheet formation was also demonstrated experimentally in a linear device ${ }^{34}$.

In recent years, theorists have shown that flux-conserving sequence of ideal MHD equilibria must develop singular 2-D current sheets. Specific examples have been calculated for reconnection in tokamak plasmas ${ }^{25}$, solar arcade models ${ }^{17,18}$, and forced reconnection. Double plasma reconnection geometry is expected to satisfy similar flux conservation constraints and, within the context of ideal MHD equilibria, develop singular current sheets. Thus, the MRX experiment will investigate how these 2-D theoretical singular current sheets are manifested in a real experiment, and it will investigate the relationship between the features of the current sheet and the third component of the merging field lines, global forcing, and the reconnection rate.

The physics of three component magnetic reconnection has been investigated by axially merging two spheromaks ${ }^{28-31}$ in TS-3, a sister device of MRX at the University of Tokyo in Japan. In these experiments, two toroidal plasma rings, with equal or opposite helicity, are formed and brought together. The MRX device ${ }^{27}$ has been built to analyze comprehensively magnetic reconnection by extending the study of this phenomena with larger sizes, higher $S$ number, and more versatile configurations.

\section{APPARATUS OF THE MRX EXPERIMENT}

Figure 2 presents a schematic of two typical operational modes of the MRX experiment, which has been designed with much flexibility and uses two flux cores to form plasmas by inductive discharges ${ }^{35}$. For example, when the external field (and hence the inward $\boldsymbol{j} \times \boldsymbol{B}$ force) is weak, the plasma is not forced away from the flux cores. Figure 2(a) shows that, in this case, the plasma assumes an annular configuration surrounding the flux cores, and reconnection driving forces are controlled by the rate at which toroidal current is induced into the annular plasmas. Thus, the reconnection rate can be studied with a variety of 
external driving forces. An alternative method to induce reconnection is to merge two spheromaks formed separately, as shown in Fig. 2(b). This configuration can be converted to yet another configuration wherein two spheromaks contact each other at a point by tilting instead of along an axisymmetric ring. Therefore, one should envision the double spheromak not as a single-reconnection experiment but as a facility capable of magnetic reconnection and turbulence studies under a variety of external driving forces.

Figure 3 shows the present MRX device. The 1.5 meter wide and 1.5 meter long vacuum vessel contains two flux cores, each with a $37.5 \mathrm{~cm}$ major radius and a $9.4 \mathrm{~cm}$ minor radius. The surface of each flux core is covered by a thin coating $(0.1 \mathrm{~mm})$ of Inconel-625, which prevents direct interaction of plasma discharges with the Epoxy-based core. The Inconel surface coat was Electro-arc spray deposited on an Alumina filled Epoxy substrate, which was applied directly onto the core overwrap. Just underneath the core overwrap is an aluminum shell conductor, which has two toroidal and two poloidal cuts and is present to help smooth flux surfaces around the core. The interior of the core consists of a bolted stack of three solid $6 \mathrm{~cm}$ thick sheets of G10 (Epoxy-glass laminate). The G10 was premachined with both toroidal and helical poloidal grooves sized to accept the $1.3 \mathrm{~cm}$ flexible cable conductors which comprise the core field windings. Inside the core, there is a 4 -turn poloidal field (PF) coil that carries a toroidal current and a helical 36-turn toroidal solenoid (toroidal field or TF coil) which can be split into two 18-turn segments. The self inductances of the 4-turn PF coil and the 36-turn TF coil are $20 \pm 2 \mu \mathrm{H}$ and $20 \pm 1 \mu \mathrm{H}$, respectively. The distance between the flux cores can be adjusted to an optimum length for each reconnection experiment. A mirror-shaped, steady-state equilibrium field (EF) field is added to keep the reconnection regime around $R=37.5 \mathrm{~cm}$. (Figs. 2 and 4 )

MRX uses up to eight $60 \mu \mathrm{F}, 20 \mathrm{kV}$ capacitors to energize each of the TF and PF coils. We have available three sets of capacitor banks each with up to $96 \mathrm{~kJ}$ of stored energy. By connecting the TF and PF coils with various tap configurations, pulsed currents of various rise times ranging from 20 to $100 \mu$ s can be drawn into both coils. By properly programming these currents, the gas is broken down via an inductive electric field and plasma 
current and internal toroidal field can be generated $^{35}$. The vacuum vessel is pumped down to $1-2 \times 10^{-6}$ Torr for the present experimental research, and the fill gas is $\mathrm{H}_{2}$.

For standard conditions $\left(n_{e}=0.1-2 \times 10^{14} \mathrm{~cm}^{-3}, T_{e}=10-30 \mathrm{eV}\right)$, MRX will have resistive, strongly magnetized plasmas. Both the electron and ion gyro-frequencies will be above their respective collision frequencies $\omega_{\text {ce }} \tau_{\mathrm{e}} \sim 130, \omega_{\mathrm{ci}} \tau_{\mathrm{i}} \sim 3$. The mean-free-path against Coulomb collisions is approximately $1-5 \mathrm{~cm}$, justifying a resistive, hydrodynamic model. Resistivity and ion viscosity will enter the energy balance equations. The MRX experiment can also be operated at higher temperatures $T_{i} \sim T_{e} \sim 30 \mathrm{eV}$, by lowering the fill pressure.

\section{MRX DIAGNOSTICS}

The MRX diagnostics consist of internal probes and noninvasive optical systems. Nonaxisymmetric structures associated with reconnection must be located and the intensity of the turbulence measured. Flows in the poloidal and azimuthal directions are expected and these also must be measured. The low temperature $(<50 \mathrm{eV})$ and short-pulsed $(<1 \mathrm{~ms})$ MRX plasma has the advantage that internal probes can be used routinely. Langmuir probes with triple pins can provide electron density and temperature data simultaneously. The plasma density measurement has been calibrated by a newly developed laser interferometer which measures the line-integrated density of the plasma ${ }^{36}$. All three components of the magnetic field can be measured during the reconnection process. To document the internal magnetic structure of the reconnection in a single shot, a 2-D magnetic probe array with grid size of $4 \mathrm{~cm}$ is placed on an $R-Z$ plane (toroidal cross-section) as shown in Fig. 4 . The following diagnostics have been installed already.

Both 1-D and 2-D arrays of magnetic probes have been used in MRX research. By integrating local magnetic field values at a specific time and in a certain toroidal plane, one can deduce a poloidal flux plot. Fine-scale probe arrays have been inserted into the current sheet region to monitor $2-\mathrm{D}$ sheet current profiles. Grid size of the internal probes varies 
from $\delta R=5 \mathrm{~mm}$ to $4 \mathrm{~cm}$ (Fig. 4). More than 20 LeCroy Model 2264 (4 MHz) digitizers are available to document evolution of the magnetic field configuration during reconnection and one Transiac Model T2001 (100 MHz) digitizer is available to explore the bandwidth of the turbulence spectrum.

Langmuir probes with triple pins can provide electron density and temperature data simultaneously. A fast sweeping technique ${ }^{37}$ has also been applied to obtain $I$ - $V$ doubleprobe characteristics with better than $10 \mu$ s time resolution. Langmuir probes (which contain double and triple pins) can map out density profiles on a single and/or multi-shot operation basis, and they can identify rapid fluctuations in density in the $1-10 \mathrm{MHz}$ frequency range near the current sheet region.

Flux loops and Rogowskii coils are used to measure global magnetic parameters, such as total plasma current and poloidal and toroidal flux. The global magnetic helicities can be deduced from these quantities with help from internal magnetic probe data.

The Mach probe ${ }^{38,39}$ is a common plasma diagnostic and is based on the same principles as a Langmuir probe. Ion saturation current is collected on back-to-back negatively biased collectors, and the difference between the two traces yields a net local ion flow velocity. Both fluid $^{39}$ and kinetic ${ }^{40}$ theories on the interpretation of the probe traces have been formulated, and we plan to adapt aspects of these theories to MRX parameters.

A retarding field energy analyzer (RFEA, or Faraday cup) has been built and installed to measure local ion temperature and plasma flows in the reconnection region. The RFEA is designed to selectively collect ion current as a function of ion energy by changing bias electric potential, thus providing information on the ion velocity distribution function. Fast sweeping of the bias potential will provide also excellent temporal resolution of ion temperature in a single plasma shot. Measurements of Doppler shifts by a spectrometer will provide information on global ion dynamics.

The line density is measured with a double pass second harmonic interferometer ${ }^{36,41}$ operating at 1.064 and $0.532 \mu \mathrm{m}$ with a sensitivity of $5 \times 10^{14} \mathrm{~cm}^{-2}$. This interferometer has a $2 \mathrm{~mm}$ diameter beam and is aligned toroidally to the MRX plasma approximately tangent 
to the major radius. The line density is measured at a single time point on each discharge. The fractional fringe shift is measured absolutely, but the results are also calibrated with respect to the pressure in a gas cell in the beam path.

\section{INITIAL EXPERIMENTAL RESULTS}

A large amount of quality magnetic reconnection data has been obtained since MRX device construction was completed in the fall of 1995 . A Lundquist number $S>700$ has been achieved already in 50-60 kA discharges, and for higher current $(100 \mathrm{kA}) S>1500$ is expected. The start-up diagnostics include magnetic and Langmuir probes, spectroscopy, flux loops, and Rogowskii coils. The initial MRX experiments have been carried out in the double annular plasma set-up in which two toroidal plasmas with annular cross section are formed independently around two flux $\operatorname{cores}^{35}$, and magnetic reconnection is driven in the quadrupole field as shown in Fig. 5. By pulsing currents in the TF coils after a quadrupole poloidal magnetic field has been established by the PF coil currents, plasmas are created around each flux core due to poloidal electric fields induced around the cores by inductive formation. At the same time, a common annular plasma forms outside the two inner plasmas which surround the two flux cores. Thus, the magnetic field domain can be divided into three sections: one public section and two private sections. The two toroidal plasmas which carry identical toroidal current with the same or the opposite toroidal field are made to merge to induce reconnection by controlling external coil currents. After the annular plasmas are created, the PF coil current can be increased or decreased. In the case of increasing PF coil current, the poloidal flux in each plasma is "pushed" toward the X-point (push mode). In the case of decreasing PF coil current, the poloidal flux in the common plasma is "pulled" back toward the X-point (pull mode), as illustrated in Fig. 5.

Typical waveforms are shown in Fig. 6 for both TF and PF coil currents. The plasma is formed around the flux cores by induction due to TF coil current from $t=200 \mu$ s after a vacuum quadrupole field configuration is established by the PF coil current. After the 
plasma diffuses into the public region of the quadrupole field (Fig. 5), "pulling" reconnection is driven during $t=260-320 \mu \mathrm{s}$. by decreasing the PF coil current. The reconnecting poloidal field is accompanied by a toroidal field (the third component), which is generated by a poloidal current in the public domain when the TF coils are connected with the same polarity (co-helicity). With the opposite polarity, no poloidal current is generated in the public domain, resulting in negligible toroidal field (null-helicity). The rise-time of the TF and PF coil currents can be varied from 20-100 $\mu$ s by changing coil connections and number of capacitors in each bank.

In the initial MRX experiments the "pull" modes have been intensively studied with and without the third vector component of the magnetic field. In both cases, identical amounts of toroidal currents are flowing parallel to each other. While similar 2-D pictures of the reconnection are expected for both cases, they are quite different in the 3-D picture, as seen in Figs. 1(i) and 1(ii). For co-helicity merging, the transition of the merging angle is gradual and smooth. But in null-helicity merging, the pitch of the field lines changes abruptly at the reconnection point. We expect strong plasma acceleration in the poloidal plane as the field lines contract after merging. This acceleration mechanism has been conjectured in the typical 2-D models.

To document the internal magnetic structure of reconnection on a single shot, a twodimensional magnetic probe array is placed in an $R-Z$ plane or toroidal cross-section, as shown in Fig. 7. Plasma parameters are as follows: $B=0.3-0.6 \mathrm{kG}, T_{e}=10-30 \mathrm{eV}$, and $n_{e}=0.5-2 \times 10^{14} \mathrm{~cm}^{-3}$. Figure 7 shows time evolution of the poloidal flux contours obtained by simply interpolating internal probe signals, assuming axisymmetry (assured by a center conductor placed in the major axis). Two distinctively different shapes of neutral sheet current layers are identified, depending on third components of reconnecting magnetic fields. Other operational conditions are held constant for each discharge. When no magnetic reconnection is induced, a typical X-shape separatrix region is observed as seen at $t=260 \mu \mathrm{s}$ in both Fig. 7(a) and 7(b). As poloidal flux are driven toward the diffusion region, a neutral sheet is formed. Without the third component (null-helicity reconnection), a thin double-Y 
shaped diffusion region is clearly identified [Fig. 7(a)]. In the presence of an appreciable third component (co-helicity reconnection), an O-shaped sheet current appears [Fig. 7(b)]. This O-point current channel grows into a spheromak configuration. To the best of our knowledge, this is the clearest experimental documentation of different current sheets, the shapes of which depend on the third component of the merging fields. The neutral current sheet in the null-helicity merging case is much narrower than in the co-helicity merging case.

It is found that merging of null-helicity plasmas occurs much faster than merging of the co-helicity plasmas, which confirms the earlier data obtained in the global plasma merging experiments on TS- $3^{28-31}$. The local features of counter-helicity merging in TS-3 are equivalent to null-helicity reconnection in MRX. A cause of the observed faster reconnection for null-helicity merging has been attributed to the effects of toroidal magnetic field field pressure. When two plasmas of parallel toroidal fields are brought together, a new equilibrium is formed among the toroidal field pressure (outward), poloidal-field pressure (attracting force), and the plasma pressure (outward). For the merging of plasmas with anti-parallel fields without the third (toroidal) field component, the attracting force becomes so dominant that reconnection is accelerated, while the toroidal field pressure slows down reconnection in the case of co-helicity merging. We note that the existence of toroidal field makes the plasma less compressible leading to a slower reconnection rate. Without toroidal field pressure in the null-helicity case, the plasma is seen to be compressed by a measured density profile which sharpens during reconnection.

The current density deduced from the measured profiles of magnetic field for the same sequence of shots provides the profile of a neutral sheet current, $j_{T}$. Fig. 8 presents a near symmetric profile of neutral sheet current induced in the null-helicity reconnection, which is always much narrower than that of the co-helicity merging case.

To measure accurately the width of the neutral sheet, a very fine scale internal probe array in which micro-scale magnetic probes are placed with $5 \mathrm{~mm}$ spacing from $R=32$ to $42 \mathrm{~cm}$ is inserted into the MRX plasma. The time evolution of $B_{Z}$ gives the radial profile evolution of the neutral sheet current, based on $j_{T} \approx \partial B_{Z} / \partial R$ since $\partial B_{R} / \partial Z \approx 0$ at the 
plane of symmetry $(Z=0)$ for null-helicity case. Figure 9 presents the radial profiles of $B_{Z}, B_{T}, j_{T}$ and pitch of field lines for co-helicity and null-helicity reconnection. In the null-helicity case, $B_{T}$ is almost zero resulting in an abrupt transition of the pitch of field lines $(\Theta)$ at the reconnection point, while in the co-helicity case, $B_{T}$ is order $B_{Z}$ resulting in a gradual change of $\Theta$ over $R$, as indicated in Fig. 1(b-i) and 1(b-ii), respectively. In the co-helicity case, the $j_{T}$ profile is broad with width of order $10 \mathrm{~cm}$. In the null-helicity case, one observes a steepening of magnetic field slope at the diffusion region and therefore a sharp neutral sheet current. The thickness of this current sheet is seen to be as narrow as $1 \mathrm{~cm}$, which is the same order as the ion gyro-radius, $\rho_{i}$, assuming $T_{i}=T_{e}$. It is observed that the thickness is inversely proportional to $B_{Z}$, which can be explained by the dependence of $\rho_{i}$ on $B_{Z}$. It is also interesting to note that $B_{Z}$ data fit very well, if not uniquely, to $B_{Z} \propto \arctan \left[\left(R-R_{0}\right) / R_{0}\right]+b\left(R-R_{0}\right)$, leading to a Lorentzian profile of $j_{T}$ $\left(\propto 1 /\left[\left(R-R_{0}\right)^{2}+d^{2}\right]+c\right)$. Figure 10 shows a typical high current discharge in the null-helicity case in which the steepening of the $B_{Z}$ profile and the sharpening of the neutral current sheet profile is clearly observed. A highly peaked density profile with a maximum value of $\sim 1 \times 10^{14} \mathrm{~cm}^{-3}$ and a moderately peaked electron temperature profile with a maximum value of $\sim 15 \mathrm{eV}$ have been measured by Langmuir probes. In the co-helicity case, relatively flat density $\left(\sim 0.5 \times 10^{13} \mathrm{~cm}^{-3}\right)$ and electron temperature $(\sim 10 \mathrm{eV})$ profiles are observed.

Since classical 2-D reconnection models do not explicitly take into account the effects of the third magnetic field component nor plasma compressibility, a quantitative comparison of the observed reconnection rate with theoretical values is not straightforward. It is observed that in the co-helicity case the reconnection velocity is three times slower than that in the null-helicity case. Quantitative comparison of experimental results to the leading theories will be carried out in the next few years of intensive research in MRX. 


\section{SUMMARY AND DISCUSSIONS}

In summary, we have constructed the MRX device to investigate the fundamental physics of magnetic reconnection in a well controlled laboratory setting. In the initial experiments, we have identified two distinctively different shapes of the diffusion region, which includes the current sheet, during co-helicity and null-helicity merging. The familiar 2-D feature, a sharp double-Y shaped diffusion regime, is identified when there is no axial magnetic field (third vector component). The thickness of this current layer is found to be on the order of the ion gyro-radius $(1-2 \mathrm{~cm})$ and decreases as we increase the magnetic field. In co-helicity merging with sizable axial field, an O-shaped diffusion region appears growing to 20-30 $\mathrm{cm}$ and the reconnection rate decreases substantially, which is attributed to the toroidal field pressure and the incompressibility of plasma. The typical speed of reconnecting flux surfaces for null-helicity merging $\left(2-3 \times 10^{5} \mathrm{~cm} / \mathrm{s}\right)$ is about three times faster than that for co-helicity merging $\left(6-8 \times 10^{5} \mathrm{~cm} / \mathrm{s}\right)$. However, both speeds are still substantially smaller than the Alfvén speed of $4-6 \times 10^{6} \mathrm{~cm} / \mathrm{s}$.

A plausible explanation for the observed difference in the shapes of the diffusion regions is as follows. A toroidal current channel is formed in the neutral sheet region during the reconnection process. Closed flux surfaces are expected to exist in the co-helicity case due to the existence of $B_{T}$. When $B_{T} / B_{Z}$ exceeds a certain value, the plasma confined in the closed flux surfaces is stable due to an absolute minimum $B$ configuration. On the other hand, flux surfaces do not exist in the null-helicity case due to the absence of $B_{T}$, inhibiting stable island growth. Even in the co-helicity case, if $B_{T}$ is small $\left(B_{T} / B_{Z} \lesssim 1\right)$, the island is unstable resulting in a thin sheet current which is driven by incoming and oppositely directed magnetic fields. Interestingly, this result is consistent with previous results obtained in an electron MHD plasma where ions were not magnetized $^{42}$.

The MRX experiment has found that magnetic reconnection depends on the merging angle of the field lines, even if the poloidal field components are kept constant. In co-helicity merging, the merging angle changes gradually through the reconnection region, while it 
changes abruptly in null-helicity merging. The quantitative dependence of the reconnection rate on the merging angle of the field lines will be investigated in the immediate future.

The results from MRX and TS-3 experiments ${ }^{29,30}$, namely that counter-helicity reconnection proceeds faster than co-helicity reconnection, are consistent with the general observation in the dayside magnetopause, in which southward IMF reconnect much faster with the earth's dipole field (northward) than northward IMF ${ }^{12,14}$. Patchy reconnection geometry, where plasmas initially contact at a point instead of along a line, has yet to be studied in a laboratory plasma. We are planning to install experimental visualization techniques aided by local 3-D probe arrays to investigate 3-D magnetic reconnection phenomena.

Although we have observed similarly structured current sheets in four toroidal locations (along the third field component) in MRX, a more detailed study is yet to be carried out to determine under what conditions symmetry breaking occurs in the structure (particularly in the toroidal direction). This study would lead to the next stage of our research: study of the dependence of the reconnection rate on global boundary conditions. In upcoming MRX research, we also plan to investigate: (1) how global MHD forces determine the profiles of neutral current sheets and/or reconnection dynamics, (2) how magnetic energy, initially released as hydromagnetic flows, is transformed into plasma kinetic energy.

\section{ACKNOWLEDGMENTS}

The authors are grateful to Mr. F. Dahlgren and Mr. D. Cylinder for their technical support. This work was jointly supported by the National Aeronautics and Space Administration, the National Science Foundation, the Office of Naval Research, and the Department of Energy.

${ }^{*}$ Present address: Univ. Tokyo, Tokyo 113, Japan

**Present address: ITER Hdq. La Jolla, CA 92037 


\section{REFERENCES}

${ }^{1}$ E. N. Parker, in Cosmical Magnetic Fields (Clarendon Press, Oxford, 1979); E.N. Parker, Astrophys. J. 180, 247 (1973).

${ }^{2}$ E. R. Priest, in Solar Magnetohydrodynamics (Ed. by P. Reidel, Dordrecht, 1984).

${ }^{3}$ V. M. Vasyliunas, Rev. Geophys. Space Phys. 13, 303 (1975).

${ }^{4}$ D. Biskamp, Physics Report 237, 179 (1993).

${ }^{5}$ R. M. Kulsrud, Phys. Plasmas 2, 1735 (1995).

${ }^{6}$ Z. Mikic, D. C. Barnes, D. D. Schnack, Astrophys. J. 328, 830 (1988).

${ }^{7}$ E. R. Priest, in Introduction to Space Physics, (ed. by G. Kivelson and C. T. Russell, Cambridge University Press, London, 1995).

${ }^{8}$ S. Tsuneta, L. Acton, M. Burner, J. Lemen, W. Brown, W. Caravalho, R. Catura, S. Freeland, B. Jurcevich, M. Morrison, Y. Ogawara, T. Hirayama, J. Owens, Solar Physics 136, 37 (1991).

${ }^{9}$ S. Tsuneta, Astrophys. J. 456, 840(1996); Astrophys. J. 456, L63 (1996).

${ }^{10}$ Y. Shi, C. C. Wu, L. C. Lee, Geophys. Res. Lett. 15, 295 (1988).

${ }^{11}$ P. Song, C. T. Russell, R. J. Fitzenreiter, J. T. Gosling, M. F. Thomsen, D. G. Mitchell, S. A. Fuselier, G. K. Parks, R. R. Anderson, D. Hubert, J. Geophys. Res. 98, 11319 (1993). ${ }^{12}$ C. T. Russell, R. C. Elphic, Geophys. Res. Lett. 6, 33 (1979).

${ }^{13}$ Y. Saito, T. Mukai, T. Terasawa, A. Nishida, S. Machida, M. Hirahara, K. Maezawa, S. Kokubun, T. Yamamoto, J. Geophys. Res. 100, 567 (1995).

${ }^{14}$ C. T. Russell, in Geophysical Monograph 90 (ed. by P. Song, B. O. Sonnerup, M. F. Thomsen, Am. Geophys. Union, Washington, 1995).

${ }^{15}$ J. B. Taylor, Rev. Mod. Phys. 28, 243 (1986). 
${ }^{16}$ M. Yamada, F. M. Levinton, N. Pomphrey, R. Budny, J. Manickam, Y. Nagayama, Phys. Plasmas 1, 3269 (1994).

${ }^{17}$ B. C. Low, Astrophys. J. 323, 358 (1987).

${ }^{18}$ B. C. Low, Astron. Astrophys. 253, 311 (1992).

${ }^{19}$ R. Matsumoto, T. Tajima, K. Shibata, M. Kaisig, Astrophys. J. 414, 357 (1993).

${ }^{20}$ P. A. Sweet, in Electromagnetic Phenomena in Cosmical Physics (ed. by B. Lehnert, Cambridge Press, New York, 1958).

${ }^{21}$ E. N. Parker, J. Geophys. Res. 62, 509 (1957).

${ }^{22}$ H. E. Petschek, "Magnetic Field Annihilation", NASA Spec. Pub. SP-50, 425 (1964).

${ }^{23}$ B. U. O. Sonnerup, D.J. Wang, J. Geophys. Res. 92, 8621 (1987).

${ }^{24}$ J. G. Hawkins, L. C. Lee, M: Yan, Y. Lin, F. W. Perkins, M. Yamada, J. Geophys. Res. $99,5869(1994)$.

${ }^{25}$ B. B. Kadomtsev, Sov. J. Plasma Phys. 1, 389 (1975).

${ }^{26}$ F. M. Levinton, S. Batha, M. Yamada, and M. Zarnstoff, Phys. Fluids B5, 2554 (1993).

${ }^{27}$ M. Yamada, H. Ji, N. Pomphrey, Y. Ono, Bull. Amer. Phys. Soc. 40, 1877 (1995).

${ }^{28}$ M. Yamada, Y. Ono, A. Hayakawa, M. Katsurai, F. W. Perkins, Phys. Rev. Lett. 65, 721 (1990).

${ }^{29}$ M. Yamada, F. W. Perkins, A. K. MacAulay, Y. Ono, and M. Katsuri, Phys. Fluids B3, $2379(1991)$.

${ }^{30}$ Y. Ono, M . Yamada, T. Akao, T. Tajima, R. Matsumoto, Phys. Fluids B5, 3691 (1993).

${ }^{31}$ Y. Ono, M. Yamada, T. Akao, T. Tajima, R. Matsumoto, Phys. Rev. Lett. 76, 3328 (1996). 
${ }^{32}$ R. L. Stenzel, W. Gekelman, J. Geophys. Res. 86, 649 (1981); W. Gekelman, R. L. Stenzel, N. Wild, J. Geophys. Res., 87, 101 (1982).

${ }^{33}$ W. Gekelman, H. Pfister, Phys. Fluids 31, 2017 (1988).

${ }^{34}$ A. G. Frank, in Plasma Physics and Plasma Electronics (Nova Science Publishers, New Jersey, 1989); S. Yu. Bogdanov, V. B. Burilina, V. S. Markov, and A. G. Frank, JETP Lett. 59, 537 (1994).

${ }^{35}$ M. Yamada, H. P. Furth, W. Hsu, A. Janos, S. Jardin, M. Okabayashi, J. Sinnis, T. Stix, K. Yamazaki; Phys. Rev. Lett. 46, 188 (1981); M. Yamada, Nucl. Fusion 25, 1327 (1985).

${ }^{36}$ N. Bretz, F. Jobes, J. Irby, "The Design of a Second Harmonic Tangential Array Interferometer for C-Mod," to be published in Rev. Sci. Instrum. (1996).

${ }^{37}$ C. Hidalgo, R. Balbin, M. A. Pedrosa, I. Garcia-Cortes, M. A. Ochando, Phys. Rev. Lett. 69,1205 (1992).

${ }^{38}$ R. J. Armstrong, D.S. Darrow, Nucl. Fusion 34, 1532 (1994).

${ }^{39}$ P. C. Stangeby, Phys. Fluids 27, 2699 (1984).

${ }^{40}$ K. S. Chung, J. Appl. Phys. 69, 3451 (1991).

${ }^{41}$ V. P. Drachev, Yu. I. Krasnikov, P. A. Bagryansky, Rev. Sci. Instrum. 64, 1010 (1993).

${ }^{42}$ R. L. Stenzel, W. Gekelman, and N. Wild, J. Geophys. Res. 88, 4793 (1983). 


\section{FIGURES}

FIG. 1. Two-dimensional (Case a) and three-dimensional (Case b) views of magnetic reconnection. When the third vector component is zero, the reconnecting field lines are exactly anti-parallel (null-helicity, Case i). With the same or opposite third field component, field lines reconnect obliquely in co-helicity (Case ii) and the reconnecting field lines are again anti-parallel in counter-helicity (Case iii).

FIG. 2. Schematic views of (a) double annular plasma configuration and (b) double spheromak configuration in MRX.

FIG. 3. Schematic view of MRX device.

FIG. 4. Cross-sectional view of MRX diagnostics. Two flux cores are located parallel to each other along a common axis. Magnetic field in the reconnection area is measured by two 2-D pick-up coil arrays. Also illustrated are toroidal and poloidal flux loops and Rogowskii coils to measure total plasma flux and current.

FIG. 5. Illustration of "push" and "pull" reconnection in the double annular plasma configuration.

FIG. 6. Typical current waveforms for both TF and PF coils energized by capacitor banks with charged voltages of $10 \mathrm{kV}$ and $8 \mathrm{kV}$, respectively. The PF coil current starts at $t=120 \mu \mathrm{s}$, generating a vacuum quadrupole field configuration until $t=200 \mu \mathrm{s}$ when a plasma is created by initiation of TF coil current with 6 mTorr filling pressure of $\mathrm{H}_{2}$ gas. The added EF field is 146 Gauss at the center of the machine. "Pulling" reconnection is driven during $t=260-320 \mu$ s by decreasing PF coil current.

FIG. 7. Time evolution of poloidal flux measured by internal magnetic probes with the same conditions shown in Fig. 6. The contours are shown only for positive values of the poloidal flux in $0.5 \mathrm{mWeber}$ intervals. By $t=290 \mu \mathrm{s}$, a double-Y shaped and O-shaped diffusion regions are formed in the (a) null-helicity case and (b) co-helicity case, respectively. 
FIG. 8. Profile of neutral sheet current in the reconnection region for null-helicity merging at $t=300 \mu$ s with the same conditions shown in Fig. 6 .

FIG. 9. Radial profiles of measured $B_{Z}$ (denoted by diamonds), $B_{T}$ (denoted by squares), field line angle $(\Theta)$ with respect to $Z$ axis, and toroidal current density across the current sheet at $Z=0$ in (a) null-helicity and (b) co-helicity cases with the same conditions shown in Fig. 6.

FIG. 10. Time evolution of measured $B_{Z}$ profiles (a) and their corresponding $j T$ profiles (b) during null-helicity reconnection with the same conditions shown in Fig. 6, except the charging voltages are raised to $12 \mathrm{kV}$ and $10 \mathrm{kV}$ for TF and PF coils, respectively, and accordingly the EF field at the center of the machine is raised to 182 Gauss.

\section{DISCLAIMER}

This report was prepared as an account of work sponsored by an agency of the United States Government. Neither the United States Government nor any agency thereof, nor any of their empleyees, makes any warranty, express or implied, or assumes any legal liability or responsibility for the accuracy, completeness, or usefulness of any information, apparatus, product, or process disclosed, or represents that its use would not infringe privately owned rights. Reference herein to any specific commercial product, process, or service by trade name, trademark, manufacturer, or atherwise does not necessarily constitute or imply its endorsement, recommendation, or favoring by the United States Government or any agency thereof. The views and opinions of authors expressed herein do not necessarily state or reflect those of the United States Government or any agency thereof. 